ORIGINAL ARTICLE

\title{
Fostering Knowledge Transfer to Strengthen the Surgical Workforce in LMIC: Lessons Learned from an International Observership Program
}

\author{
Nancy Valencia-Rojas ${ }^{1}$, Michel B Aboutanos ${ }^{2}$, Edgar B Rodas ${ }^{3}$
}

\begin{abstract}
Introduction: Virginia Commonwealth University and the Panamerican Trauma Society have worked collaboratively for 10 years in strategies focused on facilitating knowledge transfer and sharing with the ultimate goal of enhancing knowledge-base in the field of surgery, trauma, acute care, and EMS for physicians-in-training from Latin America countries. The International Observership Program (IOP) allows participants to rotate in a level 1 trauma facility and to observe all the interconnected components of a comprehensive trauma care cycle. This article describes and analyzes the limitations and lessons learned while running the IOP and offers a path for other academic institutions in high-income countries that may are interested in supporting knowledge-sharing initiatives and career development of the surgical workforce in LMICs.

Conclusion: LMIC surgical trainees can benefit from international rotations. Knowledge-sharing initiatives targeting foreign medical providers can be effective in increasing awareness of best practices for emergency and acute care and the importance of the components of comprehensive trauma systems. The goal should always be to tailor training efforts to meet the needs of LMICs and not merely to replicate the highly specialized care offered in U.S. institutions.
\end{abstract}

Keywords: Global surgery, International observership, International training experiences, Knowledge sharing, Surgical training, Surgical workforce.

\section{Resumen}

Virginia Commonwealth University y la Sociedad Panamericana de Trauma han trabajado en colaboración por 10 años en estrategias enfocadas a facilitar la transferencia y el intercambio de conocimientos con el objetivo final de mejorar la base de conocimientos en los campos de cirugía, trauma y cuidados críticos y servicios de emergencia para los médicos en formación de los países de América Latina. El Programa de Observación Internacional (POI) permite a los participantes rotar en un hospital de trauma de nivel 1 y observar todos los componentes del ciclo integral de atención de trauma. Este artículo describe y analiza las limitaciones y lecciones aprendidas durante la ejecución del POI y ofrece una guía para otras instituciones académicas en países de altos ingresos que puedan estar interesadas en apoyar iniciativas de intercambio de conocimientos y desarrollo profesional de la fuerza laboral quirúrgica en países de ingreso mediano bajo (PIMB).

Conclusión: Los residentes de cirugía de PIMB pueden beneficiarse de las rotaciones internacionales. Las iniciativas de intercambio de conocimientos dirigidas a médicos extranjeros pueden ser efectivas para aumentar la conciencia sobre las mejores prácticas en los servicios de cuidado crítico y de emergencia; y, sobre la importancia de los diferentes componentes de los sistemas integrales de trauma. El objetivo siempre debe ser adaptar los esfuerzos de capacitación para satisfacer las necesidades de los PIMB y no simplemente replicar la atención altamente especializada que se ofrece en las instituciones estadounidenses.

Palabras-clave: Observación internacional, Experiencia internacional, Educación quirúrgica, Cirugía global, Intercambio de conocimientos, Fuerza laboral quirúrgica.

Panamerican Journal of Trauma, Critical Care \& Emergency Surgery (2021): 10.5005/jp-journals-10030-1324

\section{INTRODUCTION}

Knowledge transfer or knowledge sharing "encompasses the exchange of knowledge (information, skills, experiences, or expertise) within and across organizations".' Transferring, exchanging, or sharing knowledge among surgeons in the Region of the Americas is the shared mission of the Panamerican Trauma Society (PTS) and the Virginia Commonwealth University (VCU)-Department of Surgery's International Trauma Systems Development Program (ITSDP). For 10 years, both institutions have worked collaboratively in strategies focused on facilitating knowledge transfer and sharing with the ultimate goal of enhancing knowledgebase in the field of surgery, trauma, acute care, and EMS.

In 2011, these two organizations partnered to establish the International Observership Program (IOP). The IOP is an informal hospital-based shadowing program that offers foreign-trained physicians, residents, fellows, and surgeons from LMICs the opportunity to observe a variety of patient-oriented clinical

\footnotetext{
${ }^{1-3}$ Department of Surgery, Division of Acute Care Surgical Services, Virginia Commonwealth University, Richmond, Virginia, USA

Corresponding Author: Nancy P Valencia-Rojas, Department of Surgery, Division of Acute Care Surgical Services, Virginia Commonwealth University, Richmond, Virginia, USA, Phone: +91 804828-8576, e-mail: nancy.valenciarojas@vcuhealth.org

How to cite this article: Valencia-Rojas N, Aboutanos MB, Rodas EB. Fostering Knowledge Transfer to Strengthen the Surgical Workforce in LMIC: Lessons Learned from an International Observership Program. Panam J Trauma Crit Care Emerg Surg 2021;10(2):61-65.

Source of support: Nil

Conflict of interest: None
}

procedures, academic sessions, quality improvement activities, and EMS services at VCU level 1 adult, pediatric, and burn designated

(0) The Author(s). 2021 Open Access This article is distributed under the terms of the Creative Commons Attribution 4.0 International License (https:// creativecommons.org/licenses/by-nc/4.0/), which permits unrestricted use, distribution, and non-commercial reproduction in any medium, provided you give appropriate credit to the original author(s) and the source, provide a link to the Creative Commons license, and indicate if changes were made. The Creative Commons Public Domain Dedication waiver (http://creativecommons.org/publicdomain/zero/1.0/) applies to the data made available in this article, unless otherwise stated. 
Trauma Center. The IOP's participants rotate throughout the different areas of VCU's Trauma Center including VCU Department of Surgery, Division of Acute Care Surgical Services, Trauma and Critical Care Education (CTCCE) Center, Richmond Ambulance Authority (RAA), and the VCU Center for Trauma and Critical Care Education, and more.

From 2011 to 2020, the IOP has hosted a total of 45 participants (medical students, physicians, surgery residents, trauma fellows), nationals from eleven countries including Brazil, Chile, Colombia, Costa Rica, Dominican Republic, Ecuador, Mexico, Peru, India, Sudan, and Egypt. In 2020, only two participants completed the observership during February/March. The remaining scheduled rotations were canceled due to the global COVID-19 pandemic. Given the continued risks associated with traveling abroad during the worldwide outbreak of COVID-19, the IOP made the difficult decision to put a moratorium on this program until further notice. Results of a recent survey indicated that the IOP was recognized as a valuable transfer knowledge tool where visiting physicians-intraining were introduced to novel clinical experiences and delivery care models. The results of the survey among participants showed that the biggest positive impact was that the experience expanded their view and knowledge, mostly, on the different interconnected elements of a statewide trauma care system. ${ }^{2}$ Detailed information about the IOP including activities offered during the IOP as well as demographics of the participants were described previously. ${ }^{2}$

These findings not only showed the lack of awareness of trauma systems among medical and surgical trainees from LMICs but also of the need for knowledge-sharing initiatives benefiting surgeons from LMICs. The present article describes and analyzes the limitations, opportunities, and lessons learned while running the IOP. The authors hope to offer the 10-year experience as a path for other academic institutions that are interested in supporting knowledge-sharing initiatives and career development of the surgical workforce in LMICs.

\section{Limitations, Opportunities, and Lessons LEARNED}

Although the process of establishing the IOP was relatively straightforward once the agreement between both parties was made and the terms and conditions were defined, its implementation had faced some shortcomings.

The following is a summary of the most pivotal limitations and lessons learned: Strategies to overcome them are suggested, where appropriate.

\section{Getting Buy-in from the VCU Health System Management Team}

The establishment of the IOP in VCU was relatively easy to achieve since the former Chief of the VCU Department of Surgery and the former and present Chiefs of the Division of Acute Care Surgical Services were also members of the PTS' committee that proposed this initiative. However, it was still required to get the buy-in and approval from stakeholders, such as the senior leadership and management teams of VCU School of Medicine and VCU Health System (VCUHS).

Multiple meetings were held in 2010 to highlight the benefits of building reciprocal cooperation between PTS and VCU. This turned out to be an easy sell since currently, the establishment of international academic relationships has perhaps never been simpler, nor, more necessary. There has been a rising trend among academic and professional organizations across the world seeking to foster links with global institutions. The selection of the PTS as a VCU partner was strategical and beneficial for both parties. The PTS has a strong presence in the Americas and has a successful history of collaboration with countries' professional organizations and societies to promote knowledge exchange among its members. Moreover, the PTS also hosts the annual Pan-American Congress of Trauma, Critical Care, and Emergency Surgery. Proposing a highly respectable partner with a long history was a big hit and it was welcomed without significant opposition.

Having patronage from the leadership team was essential to access the different facilities and portals that VCU offered to its students and which have overhead expenses attached to them. The enrolment of international observers required the expense of material and human resources that represented costs to the host institution. Among the direct costs included were access to electronic registration portal from the SOM, use of medical supplies (e.g., surgical scrubs and personal protective equipment); generation of the Health System photo ID card, time of administrative and clinical staff to process health and security clearance, time of clinical providers taking the time to show clinical procedures, and use of student facilities (locker, computer, shuttle, student lounge). In addition, there were also liability issues that needed to be sorted out before observers arrived related to hospital risk assessment and health and travel insurance.

\section{Suggested Strategy}

Given the undeniable financial implications and increase of workload of the hospital personnel while hosting international scholars, convincing the leadership team to buy in on this type of reciprocal knowledge exchange efforts can be, to some academic institutions a challenge. Therefore, the best strategy to reduce resistance while proposing and building a collaborative partnership is by partnering with a well-known and respected foreign professional organization or institution that brings credibility and ensures the sustainability of the initiative.

\section{Addressing the Need for Creating Opportunities for Advancement for the Surgical Workforce in LMICs}

The general objective of the IOP is to boost interest in surgical careers and to generate opportunities for career development for medical students, physicians, and junior surgeons in LMICs. It is well known that interest in surgical careers among U.S. medical students declined over the past decade. Multiple explanations have been offered for why top students are losing interest in surgical careers. One study conducted in the U.S. concluded that limited exposure to surgery during medical school may deter students from entering surgical careers. ${ }^{3}$ This drift is even more palpable in LMICs where additional issues like fewer general surgery residency programs are available; and, fellowships in trauma and emergency surgery are almost nonexistent. One study carried out among 14 countries in the Latin American Region reported that out of the total annual residency spots available $(N=30,795)$, only about $8 \%(n=2,512)$ were for the specialty of general surgery. ${ }^{4}$

The insufficient number of general surgery residency spots affects the adequacy of countries' surgeon density. An additional deterrent is the number of years that is required to complete the residency program. Surgeons require a minimum of 4 years of training as opposed to other specialties that require 2-3 years of training, for instance, the family medicine residency. Considering a 
fellowship in trauma and emergency surgery is something that only a few can aspire to pursue due to its unavailability and also financial costs. For example, fellowship in trauma and surgical critical care is not available in Ecuador. After completing the 4-5 years of residency in general surgery in Ecuador, hardly any can afford a trauma or surgical critical care fellowship in a foreign country. Unlike U.S. residents who do not have to pay for their surgery training and receive a salary enough to cover living expenses through the entire residency and fellowship programs; residents trained in LMICs must pay not only a tuition fee but also cover the majority of their annual living costs.

\section{Suggested Strategy}

The need of creating career development opportunities for physicians in training from LMICs to increase their interest in surgical specialties is evident. Similarly, the limited access of young surgeons to trauma and critical care fellowships is troublesome, and opportunities like observerships should be created. U.S. academic institutions that offer their surgical trainees international electives to practice surgery in LMICs must have moral commitment. In the name of reciprocity in global academic partnerships to create and facilitate genuine educational opportunities in the U.S. for surgeons from the countries that they visit and partner with. In 2014, the PTS established the Panamerican Trauma Society Travelling Scholarship for American trauma fellow surgeons to extend their operative trauma skills. ${ }^{5}$ This is an excellent example of bi-directional training opportunities. The experience in Colombia and the positive outcome were reported and can serve as a template for future international electives in trauma, burn, and surgical education. ${ }^{6}$ In the long-term, countries could benefit by having more surgeons offering surgical care and reducing the high burden of surgical conditions.

\section{We Expected a Higher Demand and Number of Applications}

We assumed naively that high interest in rotating in an international Level 1 Trauma Center will translate automatically in an influx of applications. We did receive a large number of online inquiries about the application process but only a few actually applied. The IOP can host two observers per month. Since the IOP is free of charge, we expected that it will be accessible to a large number of potential participants. During the first year of the IOP, we hosted only one observer. For the following 5 years, the average number of observers was 3-4 observers per year. Between 2017 and 2019, the number of participants increased to 7, 9, and 10, respectively. Although the number of participants has increased in recent years the number is below the maximum annual capacity of the IOP.

\section{Suggested Strategy}

The lower-than-expected participation is an issue that the IOP is working on improving by asking PTS members who are also faculty of medical schools to become informal local ambassadors and spread the word about the IOP in their countries. One recent assessment of the IOP reported that $63 \%$ of the former observers heard of IOP through a professor affiliated with PTS. ${ }^{2}$ Professors not only let students know about the program but also encouraged them to take advantage of this unique opportunity. In recent years, the PTS leadership team has promoted the IOP while presenting in conferences and professional meetings. This effort is reflected in a higher number of inquiries and participants. Similarly, members of the PTS leadership team live or have professional connections with countries that have higher representation in the IOP. ${ }^{2}$

\section{Only Those with Financial Resources can Participate in the IOP}

As mentioned above, the IOP hosts $<12$ international observers every year, a disappointingly low number. Some of the possible factors contributing to this are the total costs of a 2-4-week rotation for transportation and living expenses in the USA. Although the IOP is offered to PTS members at no charge, the PTS does not have financial resources to sponsor talented students and young physicians from countries with weak economies. Only those medical and surgical trainees with financial resources can participate.

Proficiency in English can be another factor that deters individuals to participate. Even though we do not reject anyone on the basics of having low English proficiency, we do encourage potential candidates to have a conversational level skill so they can understand scientific discussions about clinical cases and quality improvement, and therefore, take full advantage of this unique opportunity. Being proficient in English may also limit the participants to high-income families who are usually able to cover the cost related to English lessons. English is not spoken as a second language in many Latin American countries; this indicates that participants have to enroll in private English programs.

Since only those with financial resources to cover travel and living expenses can rotate at VCUHS, it would be ideal to have funds to support promising scholars and trainees with travel scholarships, so they can return to their communities and work in projects oriented in strengthening their local emergency and trauma systems. Unfortunately, neither the IOP nor has been able to secure funds for travel grants and this is something both the PTS and VCU should strive for.

\section{Suggested Strategy}

Academic partnerships involved in global capacity-building efforts including knowledge-sharing interventions should try to obtain funding from universities' foundations, charitable organizations, private donors, and the health care industry as a strategy to establish endowments to subsidize annual scholarships.

\section{Changing the Perception of Comprehensive Surgical and Critical Care (Trauma Systems)}

Modifying perceptions is not an easy task. Changing the perception of what comprehensive surgical and critical care means in the context of low-resource medical facilities can be undoubtedly challenging. However, it does not mean that we cannot raise awareness among the new generation of medical and surgical trainees on the benefits of having a trauma system in place. One of the specific objectives of the IOP is to foster understanding of a trauma system by offering access to a comprehensive regionalbased hospital experience under the guidance and supervision of trauma surgeons, and EMS medical directors. These kinds of experiences are useful to help medical students to model their professional pathway, narrow their specialization choices, boost research productivity in the field that is stimulated by experience, and increases knowledge in acute care surgery, and understanding of trauma care systems. ${ }^{7}$

The IOP certainly provides the fellows with a different perspective of trauma and emergency surgical care that may not be available in their native countries. Access to observe a statewide 
trauma system is a unique opportunity. A majority of international observers had never seen all the interconnected components of a comprehensive trauma care cycle including EMS dispatch and triage, hospital transfer, team activation, level 1 trauma care in an acute care facility, post-hospital care (rehabilitation services), and an injury prevention program. Past IOP participants reported that rotation at EMS was definitely a mind-changing experience, ${ }^{2}$ in particular, because U.S. EMTs are highly trained. International observers learn how paramedics take charge at the scene of an emergency and, if necessary, perform lifesaving procedures while transporting patients to the hospital, administer drugs, and, perform certain invasive procedures on patients before they arrive at the emergency department. International observers also can see how EMTs interact with police and fire department officers in cases of traffic accidents and other major emergency events.

\section{Suggested Strategy}

As surgical systems in LMICs becomes a focus of national capacitybuilding efforts, formal education on surgical systems structure in trauma should exist for the next regeneration of surgeons. ${ }^{7}$ Expanding the clinical practice perspective of a few foreign surgeons is a small part of the grand scheme of things needed to strengthen trauma systems, but it can be pivotal to ignite change in LMIC hospitals' culture and service delivery. The implementation of trauma care systems results in the improvement of patient outcomes. ${ }^{8}$ To have an efficient, comprehensive, and accountable national trauma system requires a minimum of three elements: (1) an adequate surgical density, (2) a well-trained workforce, and (3) having a basic emergency and trauma care system. The last two conditions cannot be met if local surgeons are not aware of what is needed to have a comprehensive trauma and emergency care system.

\section{Balancing Exposure to Ultra-tech Surgery While Strengthening Basic Surgical Skills}

International Observership Program's participants spend a significant amount of time in clinical areas, such as trauma bay, OR, and trauma and surgery ICU learning about advanced reanimation and surgical techniques (e.g., robotic general surgery using the da Vinci system, ultrasound) and postsurgical care. All these areas are fully staffed and equipped with state-of-the-art medical equipment, and with unrestricted access to medical supplies. Observers simply love spending time in these clinical areas but the reality is that most of them will not be able to implement some of the surgical techniques or protocols learned during their observership once they are back in their native countries. This, however, should not be a reason to hinder observers from being exposed to novel surgery procedures and equipment. This, on the contrary, has the potential to spark a passion for surgical careers in these young medical students and general physicians.

The shortage of a qualified surgical workforce represents a major challenge in LMICs. The WHO has reported that 9 out of 10 people cannot access even the most basic emergency and surgical services mostly because there are not enough surgical providers. While working in rural communities in Latin America, the current Chief of VCU Division of Acute Care Surgical Services noticed the shortage of well-trained surgical personnel. In many facilities, the few surgeons and nurses available lacked basic surgical equipment and supplies to perform essential emergency surgical procedures. During informal interactions, it became evident that many of the surgeons and nurses required additional training to deal with some of the most common general surgical emergencies, yet easy to treat, even in poor-resource settings. This is a lamentable situation, where at least $60 \%$ of the surgical operations performed in LMICs are for emergencies, including trauma and obstetrics, can be performed in primary care facilities at a low cost since staff and equipment are essentially the same. ${ }^{9}$

\section{Suggested Strategy}

The IOP hopes to contribute to this training gap by providing appropriate training to surgical personnel and strengthen their countries' essential surgical care. ${ }^{10}$ As American surgical trainees and faculty have participated in international exchanges in Latin America, U.S. academic institutions should provide valuable access to surgeons from LMICs with educational experiences in trauma and surgical systems which differ from their domestic region. This is a noble academic endeavor as the high burden of surgical diseases and injury continues because of the underdevelopment of their national systems. ${ }^{7}$

\section{Enhancing IOP Training Activities}

Although international observers are granted access to services and activities, they do not have a mentor assigned to them to oversee their overall experience. It has been proposed to match the international observers with an experienced surgeon who would be a mentor to ensure that the observers fully understand the unfamiliar process, protocols, and clinical procedures. Therefore, additional financial support for enhancing and scaling up the IOP would be ideal. Currently, funds to cover a percentage of FTE for VCU surgeons and other advance clinical practitioners have not been secured.

It is worth mentioning, however, that there are two VCU trauma surgeons (associate professors) who completed their medical school programs in their native countries in Latin America, who spend uncompensated time with the international observers. Like in any other successful project, it is always advantageous to have a champion who can commit heartily to the goal of the program, from their passion for sharing knowledge and their conviction that this opportunity can make a significant difference to the visiting scholars by expanding their horizons and boosting their career growth.

The assignment of mentors cannot be pursued without charging considerable high tuition fees as is the practice in other academic hospitals in the U.S. Billing international observers from LMICs is a strategy that is not in alignment with the knowledgesharing goals of both VCU Department of Surgery and PTS. The IOP will continue to attempt offering knowledge-sharing experiences at no cost for its observers.

\section{Suggested Strategy}

Another possible option to secure funding is to apply for training funding opportunities offered by institutions such as the Fogarty International Center which offers large grants to support collaborative research/training partnerships. Although theses desirable grants are hard to qualify and highly competitive it is still worth exploring and pursuing.

\section{CONCLUSION}

Knowledge-sharing initiatives like observerships targeting medical youth from LMIC can be effective in increasing awareness of best practices for emergency and acute care services and the importance 
of the multiple components of comprehensive trauma systems. This is backed by a previous report which stated that $89 \%$ of the IOP's participants believed that the observership helped them with their career growth. The goal, however, should always be to tailor training efforts to meet the needs of LMICs and not to replicate highlyspecialized care offered in U.S. institutions. ${ }^{11}$ Some shortcomings need to be addressed to get better outcomes. This review noted some limitations but they are by no means comprehensive. Nonetheless, there are some common limitations, lessons, and strategies that may apply to other similar IOPs.

\section{ACKnOWLedgment}

The authors would like to acknowledge Dr Rao Ivatury, past president and past executive director of PTS, and past VCU chief of Trauma Center, whose vision and impact on the development of an integrated PTS IOP at VCU is immeasurable.

\section{References}

1. Steffen Soulejman Janus. Becoming a Knowledge-sharing organization: A handbook for scaling up solutions through knowledge capturing and sharing [Internet]. Washington. DC; 2016. Available from: https://openknowledge.worldbank.org/bitstream/ handle/10986/25320/9781464809439.pdf.

2. Valencia-Rojas N, Ferrada P, Rodas EB, et al. Impact of participation in an international surgical observership program: results of an online survey. Panam J Trauma, Crit Care Emerg Surg 2019;8(3):148-153. DOI: 10.5005/jp-journals-10030-1248.
3. Peel JK, Schlachta CM, Alkhamesi NA. A systematic review of the factors affecting choice of surgery as a career. Can J Surg 2018;61(1):58-67. DOI: 10.1503/cjs.008217.

4. Organización Panamericana de la Salud. Residencias médicas en América Latina. 2011.

5. Ivatury R. Panamerican trauma society travelling scholarship: a cultural, clinical and surgical exchange. PAJTCCES 2014;3(1):1.

6. Aboutanos M, Peck GL, Paula F, et al. Can we augment the US trauma fellow's operative training? the PTS fellowship: a US surgical critical care fellow's experience in Colombia. Panam J Trauma, Crit Care Emerg Surg 2014;3(1):1-7. DOI: 10.5005/jp-journals-10030-1 077.

7. Gracias V, Gupta R, Silva JC. Nonclinical, observational trauma rotations in the United States provide international students multidisciplinary trauma program and systems education. Panam J Trauma, Crit Care Emerg Surg 2017;6(1):8-12. DOI: 10.5005/ jp-journals-10030-1165.

8. Dijkink S, Nederpelt CJ, Krijnen P, et al. Trauma systems around the world. J Trauma Acute Care Surg 2017;83(5):917-925. DOI: 10.1097/ TA.0000000000001633.

9. Prajna NV, Ravilla TD, Srinivasan S. Essential surgery: disease control priorities. Dis Control Priorities, (Volume 1) Essent Surg 2015. 1972013.

10. WORLD HEALTH ORGANIZATION Emergency \& Essential Surgical Care Programme Meeting Report WHO Global Initiative for Emergency and Essential Surgical Care Eighth Biennial Meeting. 2019.

11. Mock C, Debas H, Balch CM, et al. Global surgery: effective involvement of US academic surgery: report of the American Aurgical Association working group on global surgery. Ann Surg 2018;268(4):557-563. DOI: 10.1097/SLA.0000000000002934. 